\title{
Perihilar Bile Duct Cancer pN2 TNM
}

\section{Finding v8}

National Cancer Institute

\section{Source}

National Cancer Institute. Perihilar Bile Duct Cancer pN2 TNM Finding v8. NCI Thesaurus. Code $C 134735$.

Perihilar bile duct cancer with four or more positive lymph nodes involving the hilar, cystic duct, common bile duct, hepatic artery, posterior pancreatoduodenal, and portal vein lymph nodes. (from AJCC 8th Ed.) 